International Journal of Mechanical Engineering and Technology (IJMET)

Volume 11, Issue 9, September 2020, pp. 48-55, Article ID: IJMET_11_09_006

Available online at https://iaeme.com/Home/issue/IJMET?Volume=11\&Issue=9

ISSN Print: 0976-6340 and ISSN Online: 0976-6359

DOI: https://doi.org/10.34218/IJMET.11.9.2020.006

(C) IAEME Publication

Scope Database Indexed

\title{
EQUIVALENCE OF HIGH PAIRS ON MECHANISM
}

\author{
Odhise KOCA, Anis SULEJMANI and Drakuli LUMI \\ Mechanical Department, Faculty of Mechanical Engineering, \\ Polytechnic University of Tirana, Mother Theresa No. 4, Albania
}

\begin{abstract}
Mechanical transmissions, which are formed by links and joints, during constructive calculations, require kinematic and dynamic characteristics. The kinematics tasks of the material's point, where the transmission links are formed, are selected as below: 1. -where the material point is found, which means, its coordinate $r$ $\overrightarrow{,}$ 2. - what is the material point doing, which means, its velocity v, 3. -what will the material point do, which means, its acceleration a.

Joints and transmissions can be higher pairs, and their calculations of the kinematic characteristics becomes more difficult. Therefore, it should be used the Coriolis method with the concept of relative and carrier motion. The transition from high to low pairs, which does not use the Coriolis method in simple calculation, must be performed according to a dictionary equivalence to enter the three kinematic parameters that are mentioned above.
\end{abstract}

Keywords: vector ray, velocity, acceleration, Coriolis acceleration, relative motion, carrier, absolute.

Cite this Article: Odhise KOCA, Anis SULEJMANI and Drakuli LUMI, Equivalence of High Pairs on Mechanism. International Journal of Mechanical Engineering and Technology. 11(9), 2020, pp. 48-55.

https://iaeme.com/Home/issue/IJMET?Volume=11\&Issue=9

\section{INTRODUCTION}

\subsection{Kinematics of Point Motion in Polar Coordinates}

In case of moving a point in the plane, instead of the coordinates $x(t), y(t)$ we use the polar coordinates to determine the position of the point. We also use the directional coordinate vectors $\mathrm{r}$ and $\phi: \overrightarrow{r_{0}} ; \overrightarrow{\varphi_{0}}$. 


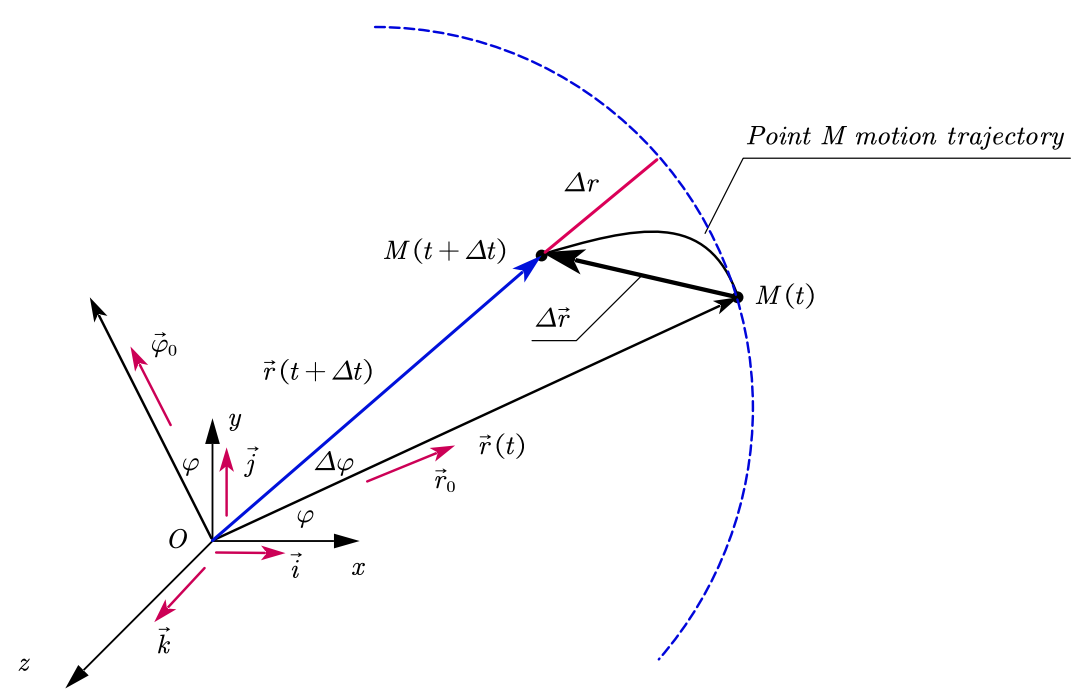

Figure 1 Kinematic parameters of the point in polar coordinates

In the direction of the radius we have the unit vector $\overrightarrow{r_{0}}$ and normal unit vector $\overrightarrow{\varphi_{0}}$ located at point $\mathrm{O}$ of the reference. Recall that $\vec{\imath}$ and $\vec{\jmath}$ are the immutable vectors of the axes Ox and Oy. The connection between them is as below:

$$
\overrightarrow{r_{0}}=\cos \varphi \cdot \vec{\imath}+\sin \varphi \cdot \vec{\jmath} \text { and } \overrightarrow{\varphi_{0}}=-\sin \varphi \cdot \vec{\imath}+\cos \varphi \cdot \vec{\jmath}
$$

We see that the vectors $\overrightarrow{\boldsymbol{r}_{\mathbf{0}}}$ and $\overrightarrow{\boldsymbol{\varphi}_{\mathbf{0}}}$ do not hold the same direction as they hold the magnitude, so they are not constant vectors so they can be derived:

$$
\begin{aligned}
& \frac{d \overrightarrow{r_{0}}}{d t}=\frac{d(\cos \varphi \cdot \vec{\imath}+\sin \varphi \cdot \vec{\jmath})}{d t}=-\dot{\varphi} \cdot \sin \varphi \cdot \vec{\imath}+\dot{\varphi} \cdot \cos \varphi \cdot \vec{\jmath}=\dot{\varphi} \cdot \overrightarrow{\varphi_{0}} \\
& \frac{d \overrightarrow{\varphi_{0}}}{d t}=\frac{d(-\sin \varphi \cdot \vec{\imath}+\cos \varphi \cdot \vec{\jmath})}{d t}=-\dot{\varphi} \cdot \cos \varphi \cdot \vec{\imath}-\dot{\varphi} \cdot \sin \varphi \cdot \vec{\jmath}=-\dot{\varphi} \cdot \overrightarrow{r_{0}}
\end{aligned}
$$

From the vector ray:

$$
\vec{r}(t)=r(t) \cdot \overrightarrow{r_{0}}
$$

From the definition of the velocity and acceleration vectors we have their expressions in polar coordinates:

$$
\begin{gathered}
\vec{v}=\frac{d \vec{r}}{d t}=\frac{d\left[r(t) \cdot \overrightarrow{r_{0}}\right]}{d t}=\frac{d r(t)}{d t} \cdot \overrightarrow{r_{0}}+r(t) \cdot \frac{d \overrightarrow{r_{0}}}{d t}=\dot{r} \cdot \overrightarrow{r_{0}}+r \cdot \dot{\varphi} \cdot \overrightarrow{\varphi_{0}} \\
\vec{a}=\frac{d \vec{v}}{d t}=\frac{d\left[\dot{r} \cdot \overrightarrow{r_{0}}+r \cdot \dot{\varphi} \cdot \overrightarrow{\varphi_{0}}\right]}{d t}=\overrightarrow{r_{0}} \cdot\left(\ddot{r}-r \cdot \dot{\varphi}^{2}\right)+\overrightarrow{\varphi_{0}} \cdot(2 \cdot \dot{r} \cdot \dot{\varphi}+r \cdot \ddot{\varphi})
\end{gathered}
$$

From the vector product of unit vectors we can write:

$$
\overrightarrow{\varphi_{0}}=\vec{k} \times \overrightarrow{r_{0}}
$$


Now we can present the kinematics expressions in vector form:

$$
\begin{aligned}
\vec{v}=\dot{r} \cdot \overrightarrow{r_{0}}+\vec{\omega} \times \vec{r} & \rightarrow \vec{\omega}=\dot{\varphi} \cdot \vec{k} \\
\vec{a} & =\overrightarrow{r_{0}} \cdot\left(\ddot{r}-r \cdot \dot{\varphi}^{2}\right)+(2 \cdot \vec{\omega} \times \dot{\vec{r}}+\vec{\varepsilon} \times \vec{r}) \rightarrow \vec{\varepsilon}=\ddot{\varphi} \cdot \vec{k}
\end{aligned}
$$

\subsection{The Kinematics of the Point in the Natural Way}

Often the trajectory of the point $\mathrm{M}$ is known from the beginning, e.g. Tirana - Durres highway when we go by vehicle, or the river bed where a river flows, or the canal where water passes for irrigation, etc. In this case we use a system of steering vectors connected to the moving point. We place the reference point on the trajectory and determine the position of the point with the arc taken on the trajectory from the reference point to the moving point $S(t)$. The trio of directional vectors are mutually perpendicular units, $\overrightarrow{t_{0}}$ directed according to the tangent, $\overrightarrow{n_{0}}$ directed according to the principal normal and $\overrightarrow{b_{0}}$ according to the binomial.

The trajectory can be replaced at the point $\mathrm{M}$ by a circle with the curvature radius $\rho$.

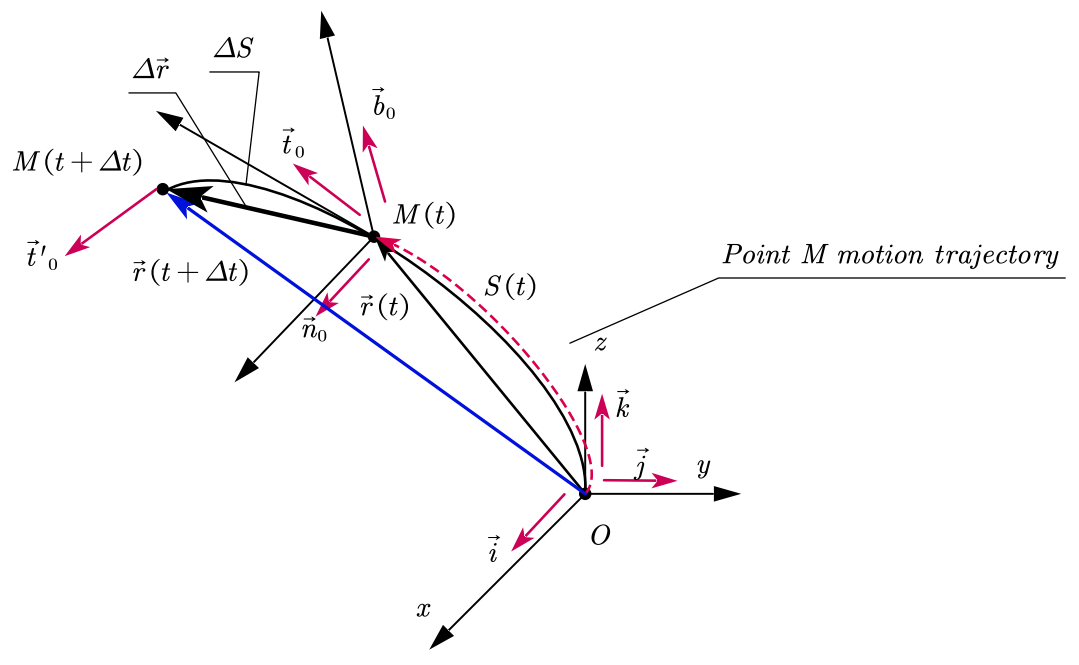

Figure 2. Kinematic parameters of the point in natural coordinates

$$
\begin{gathered}
\vec{r}=\vec{r}(S(t)) ; \vec{v}=\frac{d \vec{r}}{d t}=\frac{d S}{d t} \cdot \overrightarrow{t_{0}}=\dot{S} \cdot \overrightarrow{t_{0}} \\
\vec{a}=\frac{d \vec{v}}{d t}=\frac{d\left(\dot{S} \cdot \overrightarrow{t_{0}}\right)}{d t}=\ddot{S} \cdot \overrightarrow{t_{0}}+\dot{S} \cdot \frac{d \overrightarrow{t_{0}}}{d t}
\end{gathered}
$$

The size gets determined by the triangle of unit vectors according to the tangents for both positions:

$$
\left|d \overrightarrow{t_{0}}\right|=t_{0} \cdot \Delta \varphi=1 \cdot \Delta \varphi=\Delta \varphi
$$

while the direction is dictated by the angle that forms with the tangent:

$$
\begin{gathered}
\alpha=90^{0}-\frac{\Delta \varphi}{2} \rightarrow 90^{0}-\frac{d \varphi}{2}=90^{0} \\
\vec{a}=\frac{d \vec{v}}{d t}=\frac{d\left(\dot{s} \cdot \overrightarrow{t_{0}}\right)}{d t}=\ddot{S} \cdot \overrightarrow{t_{0}}+\dot{S} \cdot \frac{d \overrightarrow{t_{0}}}{d t}=\ddot{S} \cdot \overrightarrow{t_{0}}+\frac{\dot{S}^{2}}{\rho} \cdot \overrightarrow{n_{0}}=\overrightarrow{a_{t}}+\overrightarrow{a_{n}}
\end{gathered}
$$




\subsection{The Kinematics of the Point in the Polar-Natural Way}

We place the reference point in the center of curvature of the trajectory on this time. The trajectory is equivalent to a radius circle equal to that of the curvature. In this way the motion of the point is considered as motion according to a circle.

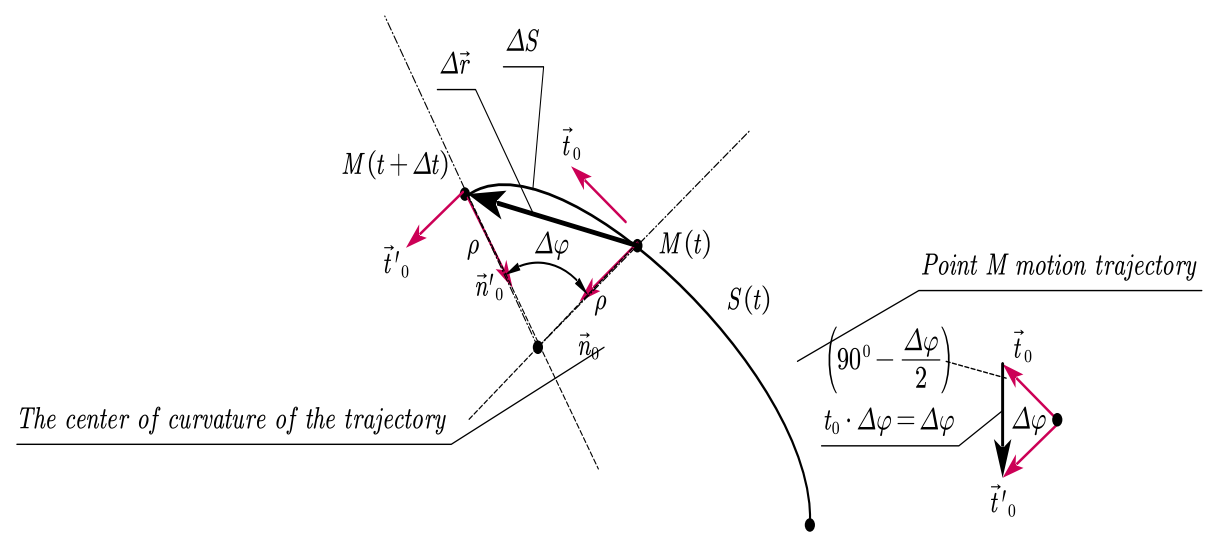

Figure 3 Natural coordinates with the polar method

For the study of point motion, we use the polar method, since the motion occurs in a plane and exactly in the main plane, consisting of the tangent and the principal normal. The polar coordinates in this case will be:

$$
\vec{r}=-\rho \cdot \overrightarrow{n_{0}^{\prime}} ; \alpha=\Delta \varphi ; \rho=\text { konst } ; \overrightarrow{r_{0}} ;=-\overrightarrow{n_{0}} ; \overrightarrow{\varphi_{0}}=\overrightarrow{t_{0}}
$$

From the analysis at these selected polar coordinates we have the kinematic expressions:

$$
\begin{gathered}
\vec{v}=\frac{d \vec{r}}{d t}=\dot{r} \cdot \overrightarrow{r_{0}}+r \cdot \dot{\varphi} \cdot \overrightarrow{\varphi_{0}}=r \cdot \dot{\alpha} \cdot \overrightarrow{\varphi_{0}}=\overrightarrow{\dot{\alpha}} \times \vec{r} \\
\vec{a}=\frac{d(\overrightarrow{\dot{\alpha}} \times \vec{r})}{d t}=\overrightarrow{\ddot{\alpha}} \times \vec{r}+\overrightarrow{\dot{\alpha}} \times(\overrightarrow{\dot{\alpha}} \times \vec{r})
\end{gathered}
$$

\section{EQUIVALENCE OF HIGH PAIRS}

High pairs are considered the couples, that meet at a point or in a line. This pair is often replaced by an equivalent link with low pairs, i.e. rotating or translatable as the case may be. High pairs as well as the translational pair allow relative motion between the joining links, i.e. the joining points do not have the same velocity and acceleration vector. The rotating pair also allows relative movement between the links, but the meet points have the same velocity and acceleration vector. Therefore, the determination of the kinematic parameters at the mechanism is facilitated.

Based on the concepts of relative motion, i.e. the Coriolis method, we have a three imaginary motions at a time:

- Absolute motion, which is the motion of the link i.e. 4, which we study, in relation to base 0, i.e. index 4.0.

- The carrier movement, which is the absolute movement of the link i.e. 3 in relation to the base 0 , the link which is connected to the link studied 4 of the high pair, i.e. index 3.0 .

- The relative motion, which is the motion of link 4 in relation to link 3, i.e. the absolute motion of link 4 when link 3 becomes the base, i.e. the index 4.3. 


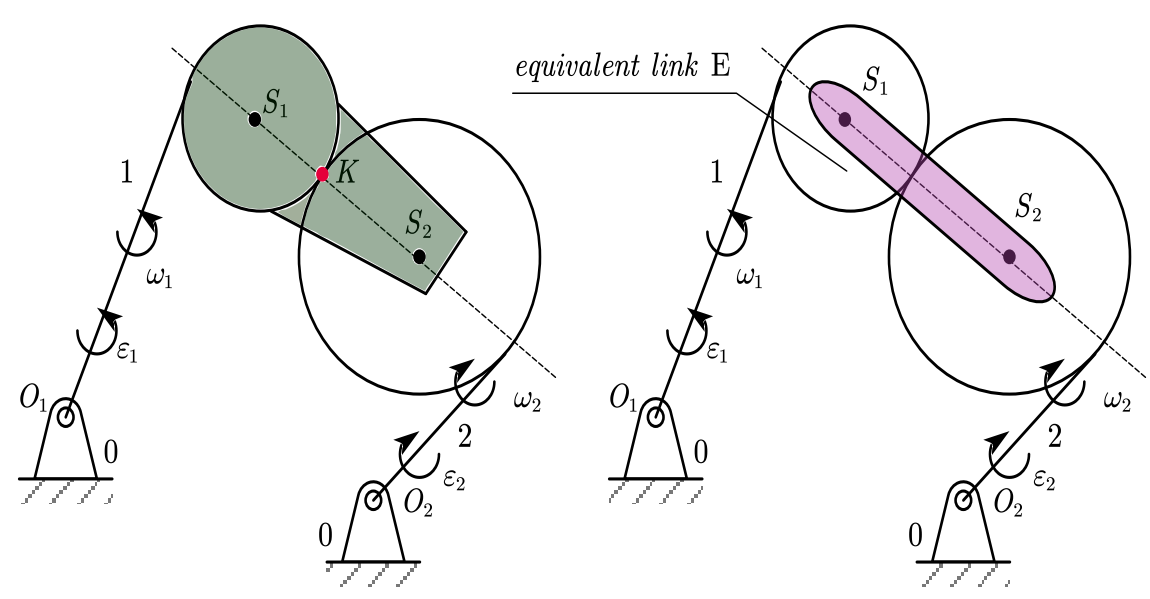

Figure 4 Node $\mathrm{K}$ as high kinematic pair

For the velocity of a point, for example S2, the Coriolis method is converted to the vector equation:

$$
\vec{v}_{S 2_{2.0}}=\vec{v}_{S 2_{1.0}}+\vec{v}_{S 2_{2.1}} \rightarrow \vec{v}_{S 2_{\text {abs }}}=\vec{v}_{S 2_{\text {bartse }}}+\vec{v}_{S 2_{\text {relativ }}}
$$

For the acceleration point such as $\mathrm{S} 4$ the Coriolis method is converted to vector equation:

$$
\vec{a}_{S 2_{2.0}}=\vec{a}_{S 2_{1.0}}+\vec{a}_{S 2_{2.1}}+2 \vec{\omega}_{1.0} \times \vec{v}_{S 2_{2.1}} \rightarrow \vec{a}_{S 2_{a b s}}=\vec{a}_{S 2_{\text {bart }}}+\vec{a}_{S 2_{\text {relativ }}}+\vec{a}_{S 2_{\text {Coriolis }}}
$$

Since the high pair allows relative motion, which is imaginary, then it can be replaced by other equivalent motions, which are also imaginary, it suffices that the vector equations of the Coriolis method do not change. This means that we will not touch the carrier motion with its characteristics, we will equate the relative motion, then the absolute motion turns out to be the same.

At the below general example $S_{2}$ and $S 1$, determined the centers of the curvature of the high pair 2-1, and $r_{2}$ and $r_{1}$ determined the curvature radius of these lines of the high pair. We analyze the relative motion of link 2 in relation to link 1 . This is easily accomplished by stopping link 1 and keeping the required relative motion clean.
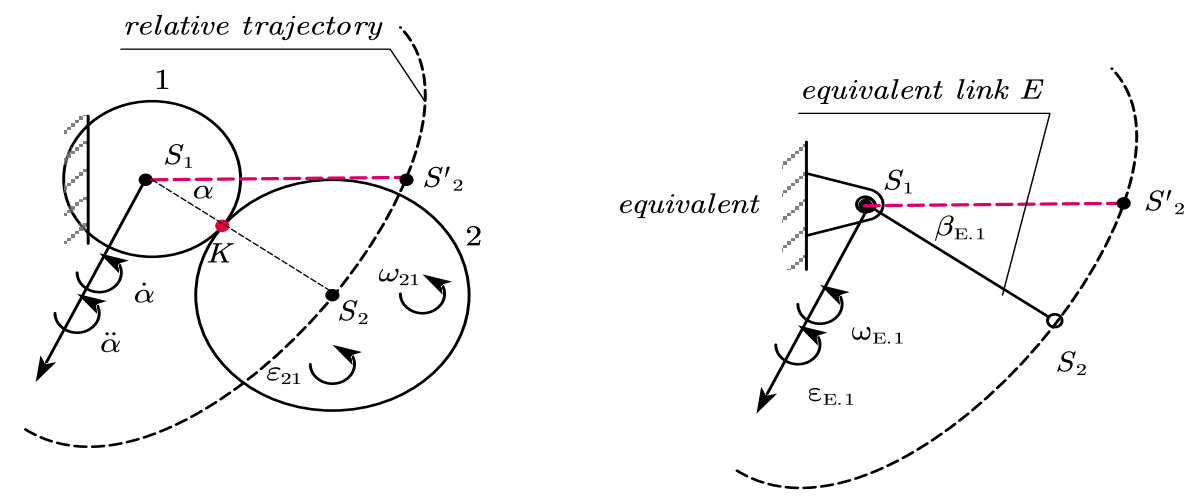

Figure 5. Relative motion of high pair links 
The center of curvature $\mathrm{S}_{4}$, in this case is a special point, which we know the trajectory, a circle with radius $r=r 2+r 1$ and with center of curvature at $S_{1}$, because it is an equidistance line from the line $\mathrm{K}_{1}$ of the curve of the link pair 2. We use the polar-natural method for the relative motion of point $S_{2}$.

$$
\begin{aligned}
& \vec{v}_{S 2_{2.0}}=\vec{v}_{S 2_{1.0}}+\overrightarrow{\dot{\alpha}} \times \overrightarrow{S_{1} S_{2}}=\overrightarrow{\omega_{1.0}} \times \overrightarrow{O_{1} S_{2}}+\overrightarrow{\omega_{E .1}} \times \overrightarrow{S_{1} S_{2}} \\
&=\overrightarrow{\omega_{1.0}} \times\left(\overrightarrow{O_{1} S_{1}}+\overrightarrow{S_{1} S_{2}}\right)+\left(\overrightarrow{\omega_{E .0}}-\overrightarrow{\omega_{1.0}}\right) \times \overrightarrow{S_{1} S_{2}}=\vec{v}_{S 1_{1.0}}+\overrightarrow{\omega_{E .0}} \times \overrightarrow{S_{1} S_{2}} \\
&=\vec{v}_{S 1_{1.0}}+\vec{v}_{S 2 S 1} \\
& \vec{a}_{S 2_{2.0}}=\vec{a}_{S 2_{1.0}}+\overrightarrow{\omega_{E .1}} \times\left(\overrightarrow{\omega_{E .1}} \times \overrightarrow{S_{1} S_{2}}\right)+\left(\overrightarrow{\overrightarrow{\varepsilon_{E .1}}} \times \overrightarrow{S_{1} S_{2}}\right)+2 \vec{\omega}_{1.0} \times \overrightarrow{v_{S 2_{21}}}=\overrightarrow{\omega_{1.0}} \times \\
&\left(\overrightarrow{\omega_{1.0}} \times \overrightarrow{O_{1} S_{2}}\right)+\left(\overrightarrow{\varepsilon_{1.0}} \times \overrightarrow{O_{1} S_{2}}\right)+\left(\overrightarrow{\omega_{E .0}}-\overrightarrow{\omega_{1.0}}\right) \times\left(\left(\overrightarrow{\omega_{E .0}}-\overrightarrow{\omega_{1.0}}\right) \times \overrightarrow{S_{1} S_{2}}\right)+\left(\left(\overrightarrow{\varepsilon_{E .0}}-\overrightarrow{\varepsilon_{1.0}}\right) \times\right. \\
&\left.\overrightarrow{S_{1} S_{2}}\right)+2 \vec{\omega}_{1.0} \times\left(\overrightarrow{\omega_{E .1}} \times \overrightarrow{S_{1} S_{2}}\right)=\vec{a}_{S 1_{1.0}}+\overrightarrow{\omega_{E .0}} \times\left(\overrightarrow{\omega_{E .0}} \times \overrightarrow{S_{1} S_{2}}\right)+\overrightarrow{\varepsilon_{E .0}} \times \overrightarrow{S_{1} S_{2}}
\end{aligned}
$$

In this relative unfolded motion, point $S_{1}$ does not move and $S_{2}$ describes a circle. Therefore, it can be replaced by a link of length $r=r 2+r 1$ and connected by a hinge at point $\mathrm{S}_{2}$.

Now we can analyze the relative motion of link 3 in relation to link 4 . This is easily accomplished by stopping link 4 and keeping the required relative motion clean see Figure 6 .

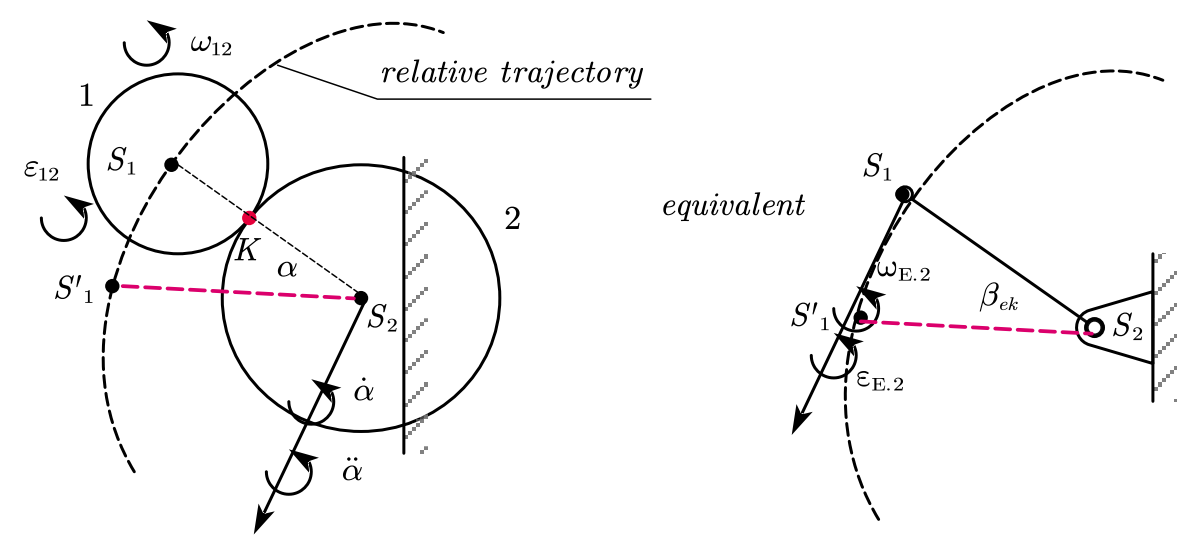

Figure 6 Relative inverse motion of high pair links

The above analysis brings us at the conclusion that relative motion is equivalent to the motion of a link of length $r=r 2+r 1$ and connected to a hinge at point $S_{2}$.

Eventually the top pair is equivalent to a new two-hinged link located in the centers of the curvatures of the lines that form the top pair.

Let us analyze the case when the top pair consists of a curve and a straight line figure 7. Equivalence is performed for relative motion only. We determine the first node of the equivalent link. We analyze the relative motion of link 1 in relation to link 2 . Link 2 becomes the base. In this case the point of contact $\mathrm{K}$ will have the trajectory of the pair line formed by link 2, i.e. circle with $S_{2}$ as a center. We analyze the relative motion of link 1 in relation to link 2. Link 2 becomes the base. In this case the center of curvature $S_{2}$ has a straight trajectory, parallel to the straight line of the kinematic pair. 
One of the equivalent node represents the hinge at the center of curvature of the curve, while the other node represents a translational node at the point of contact.

Let us analyze the case when the top pair consists of a curve and a point figure 8 . Equivalence is performed for relative motion only. Determine the first node of the equivalent link. We analyze the relative motion of link 2 in relation to link 1. Link 1 becomes the base. In this case the point of contact $\mathrm{K}$ will have the trajectory of the pair line formed by link 1, i.e. circle with $S_{1}$ as a center. We analyze the relative motion of link 1 in relation to link 2 . Link 2 becomes the base. In this case the center of curvature is the point $\mathrm{K}$. One of the equivalent node represents the hinge at the center of curvature of the curve, while the other node represents a hinge at the point of contact.

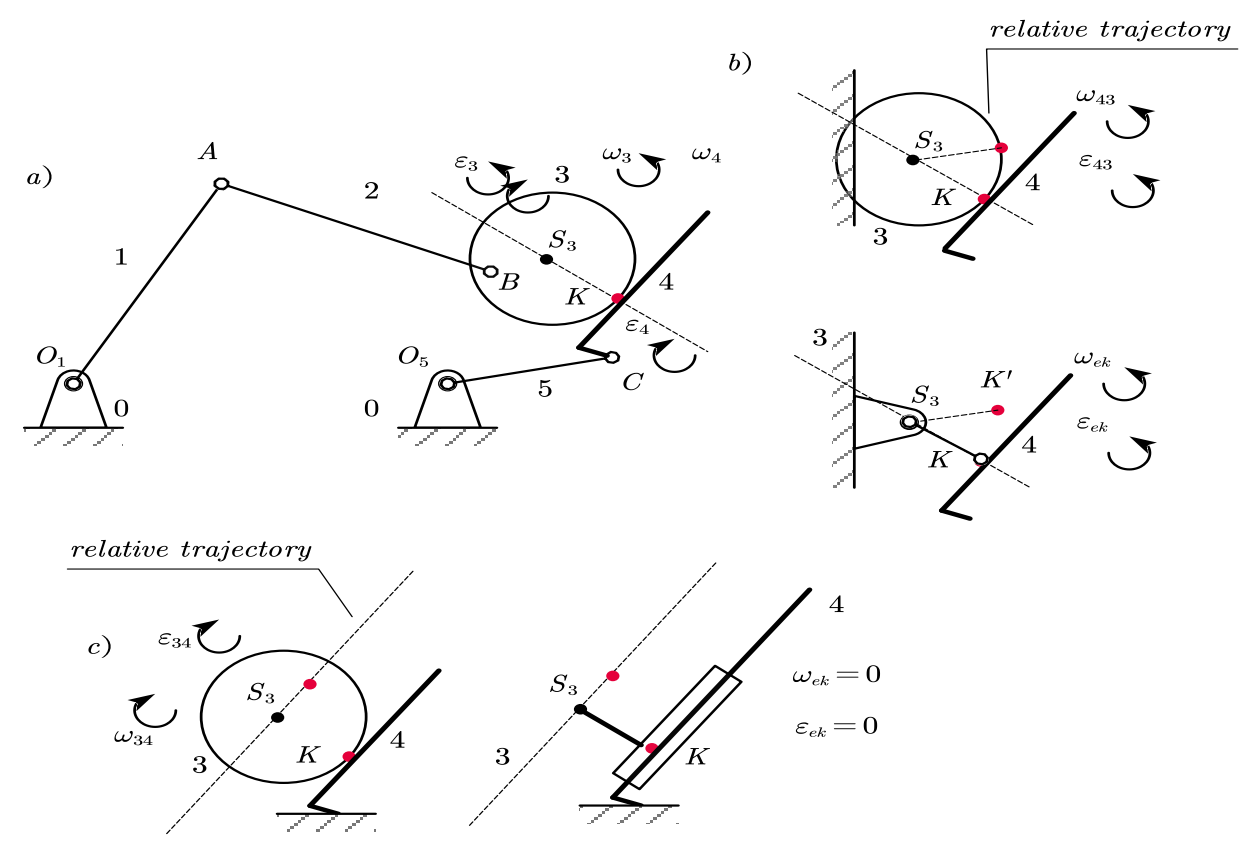

Figure 7. Equivalent transmission for high pair line-straight line
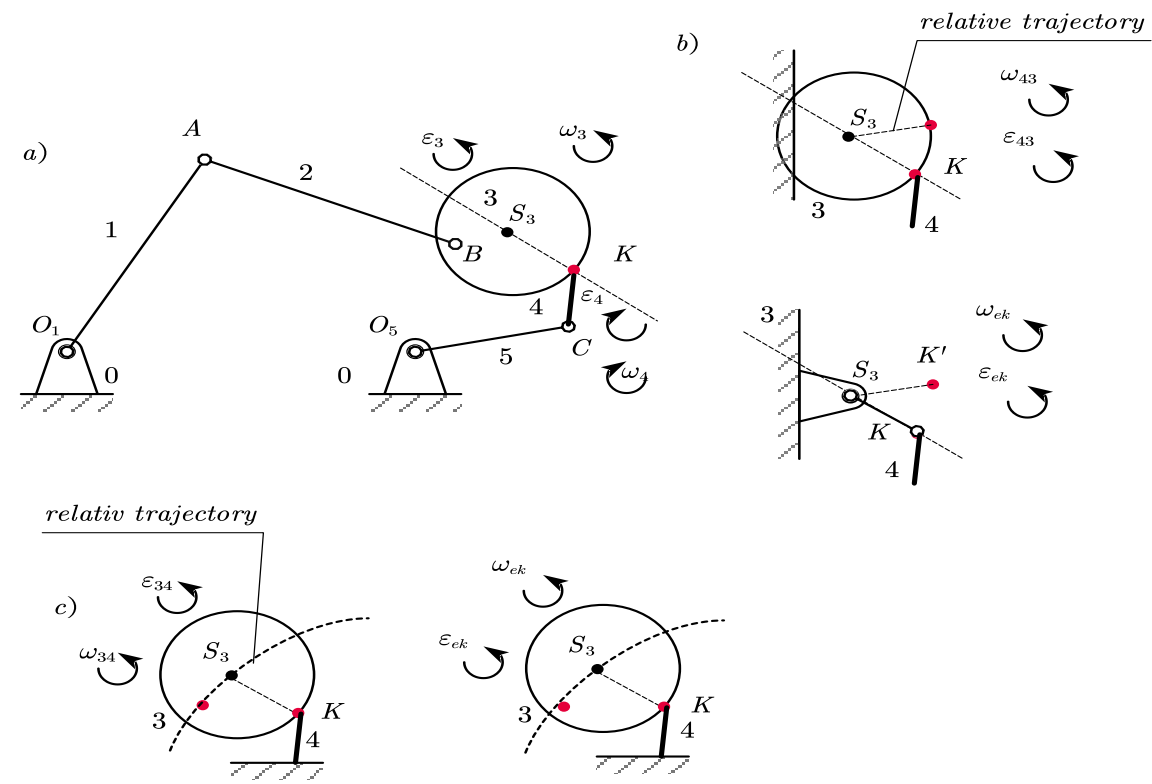

Figure 8. Equivalent transmission for high pair line-point 


\section{CONCLUSIONS}

1. Point motion can also be done with the polar-natural method.

The origin of the reference system is the center of curvature. Vector ray does not change the size and the defining vector expressions are:

2. The equivalence of the composite motion is realized only by equating its relative motion, which is an imaginary motion, and the Coriolis method remains the same.

3. High kinematic pairs or high nodes are equivalent to a two-node coin.

4. Equivalent nodes are pins at the centers of curvature for the curves.

5. Equivalent nodes are translational if the curve is represented by a straight line.

6. Equivalent nodes are magnets in case the curve is represented by a point, equivalent to a circle with zero radius.

\section{REFERENCES}

[1] Henry J. Sneck MACHINE DYNAMICS Rensselear Polytechnic Institute, PRENTICE HALL, Englewood, New Jersey 076321991

[2] Mekanikë e Aplikuar në Makina, Universiteti Politeknik Tiranë 2018.

[3] Mekanika Teorike, Kinematika, Prof.Dr. Robert Progri etj. Universiteti Politeknik Tiranë, 1989. 